\title{
¿POR QUÉ Y CÓMO DAR MIS OFRENDAS? EL MODELO DE LOS SACRIFICIOS DE PAZ
}

\author{
David Asmat, Nhilo Jaimes y Erick S. Sánchez*
}

\begin{abstract}
Resumen
Los sacrificios de paz cumplían una función especial en la experiencia devocional de los hebreos. Habiendo sido prescritos durante la estancia del pueblo israelita en el desierto del Sinaí, aún conservan principios importantes para la adoración de la iglesia actual mediante la entrega de ofrendas. En ellos se encuentran los motivos que deberían impulsar al creyente a ofrendar y la manera correcta de hacerlo. Luego de analizar de modo general Levítico 19:5, se verá que es la motivación detrás de cada ofrenda la que determina su valor. Asimismo, será notorio que el acto de ofrendar debe ser realizado sobre la base de una relación de amor a Dios y de temor a Él. Finalmente, el texto mismo revelará que una ofrenda aceptable es la que une cada aspecto mencionado anteriormente: motivación correcta, sentimiento correcto y obediencia leal.
\end{abstract}

Palabras clave: Levítico, sacrificio de paz, ofrendas, adoración

*David Asmat es Candidato a Doctor en Antiguo Testamento por la Universidad Adventista del Plata, Argentina. Profesor de la misma área en la Facultad de Teología de la Universidad Peruana Unión. Email: davidasmat@teologia.edu.pe. Nhilo Jaimes y Erick S. Sánchez son estudiantes de Teología en la Universidad antes mencionada. Emails: nhilojaimes@teologia.edu. pe, ericksanchez@teologia.edu.pe 


\title{
WHY AND HOW TO GIVE OFFERINGS? THE PEACE OFFERING MODEL
}

\begin{abstract}
The peace offerings played an important role in the devotional experience of the Hebrews. Having been prescribed during the sojourn of the israeli people in the desert of Sinai, they still conserve important principles for the current church's worship through the making of offerings. They contain the motives that should encourage the worshiper to offer and the correct manner of doing it. After analyzing in a general way Leviticus 19:5, it will be seen that is the motivation behind each offering what determines its value. Likewise, it will be notorious that the act of offering ought to be carried out on the basis of a relationship of love and fear of God. Finally, the text itself will reveal that an acceptable offering is the union of each aspect mentioned before: correct motivation, correct feeling and loyal obedience.
\end{abstract}

Keywords: Leviticus, peace offerings, worship, offerings 


\section{Introducción}

"Es mi ofrenda a Dios todo aquello con que pudiera ayudarte" (Mt 15:5), dijo un piadoso judío a su madre, de acuerdo con lo que había aprendido de los escribas en las reuniones sabáticas en la sinagoga de Genesaret. Tiempo después, dicho hombre sería testigo de una conmoción sin precedentes entre las arbóreas callejuelas de su ciudad: ¡Ha llegado el maestro de Nazaret! —era el clamor que despertaba el júbilo del gentío.

Muchos fueron curados por el Maestro, lo que causó asombro en las multitudes y en aquel judío devoto. No obstante, aún no habían sido sorprendidos lo suficiente. "Habéis invalidado el mandamiento de Dios", fueron las fuertes palabras que el Maestro pronunció ante aquella multitud, causando un dilema mental en el judío que había negado ayuda a su madre, actuando según la censurada enseńanza de sus maestros (Mt 14:34-15:6).

Este fuerte dictamen de Jesús sobre un mal uso de las ofrendas (vv. 5, 6) pudo originar una interrogante en las mentes de su audiencia ¿Acaso no era necesario y bueno separar ofrendas para Dios? De la respuesta de Jesús se desprenden dos realidades fundamentales para el asunto de ofrendar, tal cual hoy lo concebimos.

Primero, la motivación detrás de una ofrenda es la que determina su valor. Segundo, la forma correcta de ofrendar está íntimamente relacionada a la observación cuidadosa del "mandamiento de Dios" (Mt 15:6). Estas realidades expuestas por Jesús no eran —o al menos, no debían sernuevas para quienes le oían, puesto que estaban relacionadas con el concepto hallado en el Pentateuco acerca de las ofrendas y su entrega correcta. ${ }^{1}$

${ }^{1}$ La Mishná, una compilación de las leyes judías, establece que los votos de ofrendas podían ser disueltos en caso de intentar honrar a los padres. Ver W. D. Davies y Dale C. Allison, A Critical and Exegetical Commentary on the Gospel According to Saint Matthew (London, NY: T\&T Clark International, 2004), 2:523. No obstante, en el tiempo de Jesús, los escribas y fariseos consideraban que el voto de ofrendas debía cumplirse indefectiblemente, y que dicho voto anulaba el deber asistencial de los hijos para con sus padres (Mt 15:5). Aunque esta concepción tenía cierta base en el Pentateuco ( $\mathrm{Nm}$ 30:2), era inadecuada en su aplicación puesto que pasaba 
Un caso modelo puede ser el de los sacrificios de paz. De acuerdo con lo ordenando, quien ofreciera tales sacrificios a Dios era guiado con estas palabras: "ofrecedlo de tal manera que seáis aceptos" (Lv 19:5). Hay un doble significado comprendido en este precepto, el cual refleja la declaración de Jesús en Genesaret. En primer lugar, la sola mención de los sacrificios de paz alude a la noción que se tenía sobre la ocasión y el motivo para ofrecerlos. ${ }^{2}$ En segundo lugar, es destacable que existía una norma que determinaba la aceptación de la ofrenda. ${ }^{3}$

Así pues, del texto surgen tres preguntas inevitables de las que se ocupará este artículo: ¿Cuáles son los motivos para ofrecer un sacrificio de paz? ¿Qué determina una ofrenda aceptable? ¿Qué principios contenidos en aquellos ancestrales sacrificios son aplicables a la fidelidad de la iglesia del siglo XXI?

\section{Levítico 19: el texto y su contexto}

Mientras el pueblo de Israel se hallaba acampando en la llanura

por alto que también existían prescripciones que contemplaban casos especiales, evitando que se lleguen a extremos innecesarios (Nm 30:3-16). En el caso de la confrontación de Jesús con los escribas y fariseos de Mateo 15:1-9, se destaca la preponderancia que la tradición tenía sobre el mandamiento de Dios, lo cual fue desaprobado por el Maestro. Esto se debe a que se buscaba una excusa para evadir el quinto mandamiento amparándose en una tradición que sostenía que todo lo consagrado para el Templo quedaba exento de uso para otros quehaceres, aun si éstos fueran justos. Ver Matthew Henry, Comentario Bíblico de Matthew Henry, trad. Francisco Lacueva (Barcelona: Editorial CLIE, 1999), 1136.

${ }^{2}$ Debido a lo estipulado en Levítico 7:12-17 y 22:18-30, es posible afirmar que cada vez que se ofrecía un sacrificio de paz debían tenerse en cuenta razones que denotaban las ocasiones requeridas para el sacrificio.

${ }^{3}$ Esta realidad se halla implícita en el texto hebreo. El uso del término hebreo rāṣôn junto a la preposición $l$ equivale a "de modo aceptable" o "válidamente". Cualquiera de estas traducciones transmite por inferencia la idea de una norma que hace aceptable o válida alguna cosa. Ver Luis Alonso Schökel, "רָצָ", en Diccionario bíblico hebreo-español (Madrid: Editorial Trotta, 1994), 717. 
de Er-Raha, en el desierto sinaítico ${ }^{4}$ (Éx 19:1), en el año 1446 a.C., ${ }^{5}$ Moisés recibió diversas instrucciones concernientes a la vida del pueblo y al santuario. ${ }^{6}$ Allí también se definieron por primera vez los distintos sacrificios y ofrendas que se realizarían en el tabernáculo y se establecieron procedimientos para ellos. ${ }^{7}$ Una clase especial de ofrendas eran los sacrificios de paz, para los que hay textos prescriptivos y descriptivos ${ }^{8}$ en todo el Pentateuco -y aún fuera de él- que muestran de modo integral su concepto. ${ }^{9}$ Sin embargo, es el libro de Levítico el que de forma

${ }^{4}$ H. D. M. Spence y Joseph S. Exell, eds., The Pulpit Commentary: Exodus Vol. II, (London: Funk \& Wagnalls Company, 2004), 103.

${ }^{5}$ Jack Finegan, Handbook of Biblical Chronology (Peabody, Massachusetts: Hendrickson Publishers, 1998), 225-228.

${ }^{6}$ Las prescripciones pertinentes al santuario se sugieren desde Éxodo 19, al mostrar el deseo de Dios para su pueblo: "me seréis un reino de sacerdotes y de gente santa" (Lv 19:6). De modo específico, las ordenanzas para la construcción del santuario son registradas desde Éxodo 25 hasta el 31; y la ejecución de la obra se detalla en los capítulos 35-40. El libro de Levítico inicia cuando Jehová, luego de haber descendido al tabernáculo ya construido (Éx 40:34-38), llama a Moisés ( $\mathrm{Lv} 1: 1$ ) para explicarle todo lo concerniente a los procedimientos rituales del santuario y otras normas relacionadas con la santidad del pueblo. Así, Éxodo indica el lugar donde Dios debe ser adorado, mientras que Levítico enfatiza cómo debe ser adorado. Ver Ver Mark F. Rooker, Leviticus, de The New American Commentary, ed. E. Ray Clendenen (Nashville, TN: Broadman \& Holman Publishers, 2000), 3:39. En adelante NAC.

${ }^{7} \mathrm{El}$ rasgo breve y sencillo del ritual sacrificial hebreo prácticamente no tiene parangón en todo el Antiguo Cercano Oriente, salvo en Ugarit. En Egipto y Emar, por ejemplo, la duración y los elementos incluidos en un ritual superaban por mucho a la práctica hebrea. Por otro lado, el calendario festivo-ritual anual de los hebreos es una pequeñez frente a sus contrapartes mesopotámicas. Ver K.A. Kitchen, On the Reliability of the Old Testament (Grand Rapids, MI: Eerdmans, 2003), 281.

${ }^{8}$ Los textos rituales prescriptivos se refieren a la norma a seguir mientras que los descriptivos narran la ejecución de aquella norma. Ver Gerald Klingbeil, "La perla perdida (o escondida) del Pentateuco: relevancia, significado y función del ritual bíblico", en Volviendo a los Origenes: entendiendo el Pentateuco, ed. Merling Alomía, et al (Lima: Editorial Imprenta Unión, 2006), 225-231.

${ }^{9}$ Las menciones de sacrificios de paz en el Pentateuco se hallan con más abundancia en Levítico, en el cual se detallan los procedimientos y la ejecución de ellos. La ocasión que se requería para un sacrificio de paz puede percibirse en los históricos (el sacrificio de paz como 
particular y pormenorizada aborda la forma, la idoneidad, y la santidad de tales sacrificios. ${ }^{10}$ Así pues, todo ofrecimiento de sacrificios de paz se regía por las siguientes palabras: "Y cuando ofreciereis sacrificio de ofrenda de paz a Jehová, ofrecedlo de tal manera que seáis aceptos" (Lv 19:5). ${ }^{11}$ Tal precepto forma parte de una lista más amplia cuya consigna principal es "santos seréis, porque santo soy yo Jehová vuestro Dios" (Lv 19:2). Con esto, Dios exhortó a Israel a ser santo y, a continuación, le explicó la forma de conseguir aquella santidad incluyendo en sus declaraciones la reglamentación para el sacrificio de paz.

\section{Razón y motivación: ¿Por qué ofrecer una ofrenda de paz?}

La frase "y cuando" ( $\left.w^{e} \underline{k} \hat{\imath}\right)$ introduce el texto de Levítico 19:5 y, al mismo tiempo, inserta la noción de tiempo en el texto que le sigue: "ofreciereis sacrificio"12 de ofrenda de paz a Jehová”, aludiendo a las ocasiones

expresión de gratitud de Manasés luego de haber sido librado, 2 Cr 33:16), en los sapienciales (relacionados con la realización de votos, $\operatorname{Pr} 7: 14$ ), y en los proféticos (mencionados luego de todos los demás sacrificios, Ez 43:27).

${ }^{10} \mathrm{El}$ sistema sacrificial hebreo es descrito en su mayor parte en Levítico, razón por la cual se ha llegado a llamarlo "el libro de la ley por excelencia". Las leyes levíticas regulan principalmente la adoración a Dios por parte de Israel, y el quehacer sacerdotal. Ver R. Laird Harris, Leviticus, de The Expositor's Bible Commentary, ed. Frank E. Gaebelein (Grand Rapids, MI: Zondervan, 1990), 501.

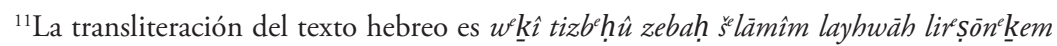

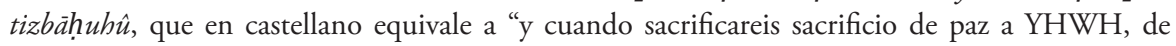
modo aceptable a ustedes los sacrificaréis".

${ }^{12}$ Literalmente, "sacrificareis sacrificio de paz a YHWH". El uso conjunto y yuxtapuesto del verbo zäbah (sacrificar) y su forma nominal zebah (sacrificio), se refiere siempre en el contexto del Antiguo Testamento a un sacrificio de ganado vacuno, caprino u ovino, el cual se realizaba para crear comunión entre el Dios a quien se dirigía la ofrenda y quien o quienes la ofrecían. Por otro lado, este mismo sentido del verbo puede hallarse en términos similares en ugarítico, fenicio, púnico y árabe. Véase Richard E. Averbeck, "חָזָז", en New International Dictionary of Old Testament Theology and Exegesis, ed. Willem A. VanGemeren (Grand Rapids, MI: Zondervan, 1998) 1:1066-1068. 
en que se realizaban tales ofrendas. Se esperaba que los sacrificios de paz sean realizados en tres ocasiones que requieren, a su vez, tres razones. En primer lugar, estos eran realizados 'al tôdāh o "en acción de gracias" (Lv 7:12): por los actos benevolentes de Dios en favor de una persona (2 S 6:17-18), o del pueblo en general (Lv 9:18). No obstante, el texto implica algo más que gratitud, dado que la expresión hebrea 'al tôdāh equivale en su sentido más básico a "para confesión" o "para alabanza”. ${ }^{13}$ En consecuencia, la primera razón por la que se ofrecía un sacrificio de paz incluye un doble aspecto relacionado al agradecimiento: confesión de pecados (2 S 24:25) y alabanza a Dios (2 Cr 33:16). ${ }^{14}$

En segundo lugar, se realizaban en cumplimiento de un neder o "voto" (Lv 7:16), es decir, una promesa previa de parte del oferente (Pr 7:14). Dicho voto estaba regido por principios que pueden hallarse fuera de contextos sacrificiales. De este modo, se sabe que al hacer un voto (neder) una persona demandaba que Dios actúe en su favor y, para conseguirlo, no solo prometía bienes (Lv 27:14), sino también adoptar cierto estilo de vida por determinado tiempo o perpetuamente (Nm $6: 2,3) .{ }^{15}$ Por otra parte, se necesitaba seriedad al hacer un voto a Jehová pues "lazo es al hombre hacer apresuradamente voto" ( $\operatorname{Pr} 20: 25)$ y si no lo cumpliese "ciertamente lo demandará Jehová” de él (Dt 23:21).

${ }^{13}$ En realidad, no existe término hebreo que se corresponda de manera exacta con el vocablo castellano "gracias". El término proviene de una raíz cuyo abanico semántico se aplica en: 1) confesión del pecado, 2) confesión de adoración, y 3) elogio a un hombre. De ese modo tôdāh llega a significar fundamentalmente "confesión". Véase, Ralph. H. Alexander, "הדָָ", en Theological Wordbook of the Old Testament, ed. R. Laird Harris (Chicago: Moody Press, 1980), 364-365. En adelante TWOT.

${ }^{14}$ Aunque en su aplicación las ofrendas de paz estaban ligadas a la confesión del pecado, su función era distinta a la desempeńada por las ofrendas por el pecado (Lv 4:1-5:13), por la culpa (Lv 5:14-6:7), y el holocausto (Lv 1). Normalmente, cuando las ofrendas de paz eran de confesión del pecado se habrían realizado después de todos los otros sacrificios (Lev 9:22).

${ }^{15}$ Marburg-Cappel O. Kaiser, "נָָּרָ", en Theological Dictionary of the Old Testament, eds. G. Johannes Botterweck, Helmer Ringgren y Heinz-Josef Fabry, trad. David E. Green (Grand Rapids, MI: Eerdmans, 1998), 9: 244. En adelante TDOT. 
En tercer lugar, un sacrificio de paz se ofrecía también como un zebaḥ n'däbāh o "sacrificio voluntario" (Lv 7: 16). Para distinguir una ofrenda voluntaria de una hecha por voto ${ }^{16}$ han de considerarse las situaciones subyacentes. Mientras que en el primer caso se requiere la respuesta de Dios para cumplir el voto -pues éste demanda condiciones-, el énfasis en el segundo caso recae enteramente en la espontaneidad o libre voluntad de quien ofrenda. ${ }^{17}$ Una revisión de estas tres razones por las que se ofrecía un sacrificio de paz establece los motivos que generan -o deberían generar- el desprendimiento en alguien que ofrenda: gratitud expresada en confesión y alabanza, cumplimiento de pactos y ofrecimiento de modo incondicional. Queda claro que estas motivaciones por sí solas no le daban al adorador la experiencia excepcional del sacrificio de paz. Algo más debía tenerse en cuenta.

¿Para quién y dónde doy mis ofrendas? La frase "a Jehová" está relacionada con los motivos expuestos arriba e indica a quién se dirigía todo sacrificio de paz. ${ }^{18}$ Cada uno de ellos conducía a una persona a ofrendar y, a su vez, cada ofrenda era hecha "a Jehová". De este modo, oferente, ofrenda y recipiente estaban inseparablemente unidos en una acción simbólica cuya significancia estaba determinada por las motivaciones iniciales: gratitud, cumplimiento de pactos y entrega incondicional.

${ }^{16}$ La complejidad de establecer esta distinción radica en la motivación similar que hay al hacer un voto de ofrendar y ofrendar de manera voluntaria, ya que ambas cosas dependen de la voluntad del sujeto. Además de ello el uso adverbial de $n^{e} d \bar{a} b \bar{a} h$ afectando el verbo dib-bar tā en Dt 23:23 intrinca más el asunto. Ver Ibíd., 221-222.

${ }^{17}$ Nobuyoshi Kiuchi, "Spirituality in offering a peace offering", Tyndale Bulletin 50/1 (1999): 25 .

${ }^{18}$ En hebreo se lee layhwāh. La preposición $l$ afijada al nombre divino funciona como una lamed (letra equivalente a $l$ en el hebreo) de "interés" o "ventaja", cuya función es señalar a la persona a quien es dirigida la acción, tal como un objeto indirecto en castellano. Ver Bruce K. Waltke y M. O'Connor, An Introduction to Biblical Hebrew Syntax (Winona Lake, IN: Eisenbrauns, 1990), 207. 


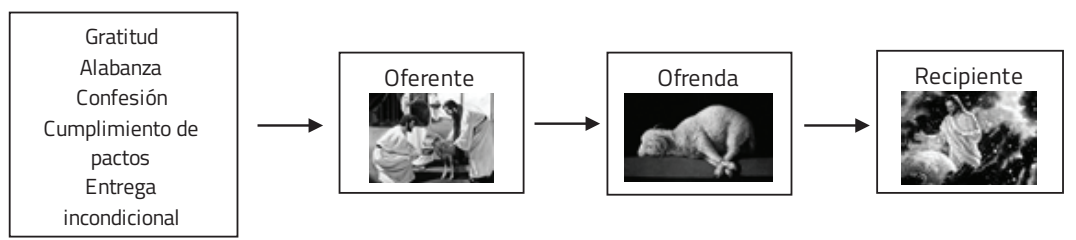

Figura 1. Relación de los elementos en un sacrificio de paz

Esta realidad es enfatizada por el uso del nombre propio divino "Jehová", el cual presenta a Dios en la esfera de su carácter misericordioso y amante. Así queda definido el carácter relacional de un sacrificio de paz, es decir, que la ofrenda era dada sabiendo a quién iba dirigida y con todo el sentimiento que ello involucrara. ${ }^{19}$ Por otra parte, cabe considerar un aspecto más de tales sacrificios. En Levítico 3:1 se registra una regulación que prescribe ofrecer sacrificios de paz "delante de Jehová". ${ }^{20}$ Esta frase contiene un sentido espacial, señalándole al adorador que estaba en la presencia de Jehová y ante su mirada examinadora. ${ }^{21}$ Con esta percepción en mente -a saber, que el sacrificio era hecho a Jehová y delante de él- se esperaba que el pueblo ofrendase con reverencia y temor a Dios en el santuario en que moraba su presencia.

El sencillo argumento de esta sección es que ofrecer un sacrificio de paz portaba y, a decir verdad, requería, dos aspectos en su ejecución: el aspecto relacional, que incluía saber que la ofrenda era entregada a Jehová

${ }^{19}$ Basado en una comparación del hebreo $s^{r} l$ mîm con el acadio šulm nu, Levine sugirió una conexión cercana entre la ceremonia pactal en ocasión de la entronización de Saúl en Gilgal (1 S 11:15), y un paralelo del ACO, a saber, la "ofrenda tributaria" que el rey Pabil presentó a Keret para hacerle levantar el asedio. No obstante, el motivo de los sacrificios de paz bíblicos difiere de sus paralelos extrabíblicos en que los primeros eran presentados al Señor, YHWH. Richard E. Averbeck, "Sacrificies and offerings", en Dictionary of the Old Testament: Pentateuch, eds. T. Desmond Alexander y David W. Baker (Downers Grove, Ill: InterVarsity, 2003), 715-716.

${ }^{20} \mathrm{La}$ traducción literal del hebreo lipenê y̌hw h es "en el rostro de Jehová".

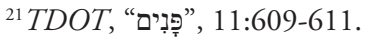


¿Por qué y cómo dar mis ofrendas?

El modelo de los sacrificios de paz

por razones fundamentadas en la respuesta humana a los actos divinos; y el aspecto espacial, que involucraba la reverencia inspirada por el santuario, concebido como la morada de Dios, y por la noción de estar ante la misma presencia divina. Estos principios, como se verá más adelante, son importantes cuando se consideran las ofrendas del creyente en estos días.

\section{Alcanzar la aceptación: ¿Cómo ofrecer un sacrificio de paz aceptable?}

El tema principal de Levítico 19:5 puede definirse certeramente por el verbo que marca su principio y fin: "sacrificareis" y "sacrificaréis". ${ }^{22}$ El primero se refiere al pueblo de forma directa; el segundo, al pueblo junto con los sacrificios de paz. Así, el actor y la acción constituyen el punto focal. Esta misma realidad se encuentra en el término empleado para designar lo que Dios esperaba de una ofrenda, a saber, que fuera ofrecida lir Șôn ${ }^{e} k e m,{ }^{23}$ "de modo que sean aceptadas en favor de ustedes". ${ }^{24}$ Solo en esta corta expresión están involucrados dos conceptos.

El primero tiene que ver con la idoneidad del sacrificio de paz, la

${ }^{22}$ Tal como se traduciría directamente de los términos hebreos tizbệu y tizbāḥhhu. Esta peculiar presentación del versículo puede indicar que el versículo sigue un esquema en quiasmo definido por el verbo zābah. Ver John E. Hartley, Leviticus, Word Biblical Commentary, ed. Bruce M. Metzger, David A. Hubbard y Glenn Baker (Dallas, TX: Word Books, 1992), 4: 304.

${ }^{23} \mathrm{El}$ término hebreo rāṣ̂n diferencias considerables de significado. Sin embargo, siempre que aparece en un contexto cúltico o ritualístico llega a denotar aceptación y el estado agradable de algo o alguien respecto a otro. Ver Éx 28:38; Lv 1:3; 19:5; 22:19; 2:20; 22:21; 22:29; 23:11; Pr 15:8; Is 56:7; Is 60:7; Jer 6:20; Mal 2:13. Existen otros ejemplos con el mismo significado en la literatura poética (Sal 19:15) y sapiencial (Pr 10:32; 11:1; 11:20;12:22; 15:8) donde el elemento cúltico no está incluido.

${ }^{24} \mathrm{La}$ preposición $l^{e}$ y el sufijo plural en segunda persona $\mathrm{kem}$, requieren incluir en la traducción a los sacrificios de paz y al pueblo. Considerando esto puede hacer la traducción al inglés así: "in such a way that it will be accepted on your behalf." Ver $N A C, 3: 255$. Por otro lado, el talmud babilónico registra "no por mi bien vosotros sacrificáis sino por el vuestro". Ver B. Talmud Mena ot 110a, citado en Jacob Milgrom, Leviticus 17-22: A New Translation with Introduction and Commentary, Anchor Bible (New Haven, London: Yale University Press, 2008), 1620. 
cual es descrita en el versículo siguiente (Lv 19:6). El pueblo fue instruido sobre este asunto de esta forma: el sacrificio de paz "será comido el día que lo ofreciereis", refiriéndose a la ofrenda por acción de gracias que obedecía a este requerimiento ( $\operatorname{Lv} 7: 15)$. "El día siguiente" se comerían solo las ofrendas votivas y voluntarias ( $\operatorname{Lv} 7: 16)$, "y lo que quedare para el tercer día, será quemado en el fuego" (Lv 19:6). En este precepto destaca el elemento de confraternización de un sacrificio de paz, dado que el pecho y la espaldilla derecha del becerro, cordero o cabra que se ofreciese (Lv 3:1, $7,12)$, eran compartidos con los sacerdotes $(\operatorname{Lv} 7: 29-36),{ }^{25}$ y el resto con los amigos o familiares invitados del oferente. ${ }^{26}$

El segundo, muestra lo singular que era este tipo de sacrificio, puesto que era el único que permitía manipular el cuerpo del animal sacrificado al que ofrendaba y, de este modo, una persona recibía en retorno parte de aquello que entregaba a Jehová. En armonía con esto, el término lirệō$n^{e} \underline{k} e m$, "de modo aceptable a ustedes", adquiere su sentido más pleno como indicador de aceptación por parte de Jehová, lo que, a su vez, redunda en beneficio para el adorador. Ahora bien, este par de conceptos ayudan a determinar lo que hacía aceptable un sacrificio de paz, dando una respuesta clara: seguir los lineamientos establecidos en la ley, ${ }^{27}$ de otro modo, "si se comiere el día tercero, será abominación; no será acepto” (Lv 19:7).$^{28}$

Una vez que se ha comprendido esto se puede avanzar un paso más en la comprensión de un sacrificio de paz idóneo, al responder la pregunta ¿qué principio trascendental estaba contenido en la ley para los

\footnotetext{
${ }^{25}$ Además de esto, el sacerdote recibía las tortas de harina que presentaba el oferente como "ofrenda mecida" (o ťnûfäh). Véase, Anson Rainey, "Sacrifice", en Encyclopaedia Judaica (Jerusalén: Keter Publishing House, 1996), 14: 604.

${ }^{26}$ W. Gunther Plaut, ed., The Torah: A Modern Commentary (New York: Union of American Hebrew Congregations, 1981), 765.

${ }^{27}$ Esta verdad es enfatizada por el uso de hill-lēl (profanó) en Lv 19:8. Se emplea el término para denotar violencia contra la ley de Dios y contra su pacto (Sal 55:20; Sof 3:4).

${ }^{28} \mathrm{El}$ término castellano esta traducido de una raíz hebrea que alude a las carnes inmundas o en estado de descomposición (Is 65:4; Ez 4:14).
} 
sacrificios de paz? El ritual ordenado por la ley era similar al de los otros tres tipos de sacrificios: los holocaustos ( $\mathrm{Lv} 1)$, los sacrificios por el pecado (Lv 4:1-5:13) y por la culpa (Lv 5:14-6:7). ${ }^{29}$ Sin embargo, su función era distinta. Mientras que para estos tres últimos sacrificios se registran claramente sus propósitos perdonadores y expiatorios, para el sacrificio de paz no hay una declaración directa. Con todo, el propósito de éste queda definido por dos factores: primero, el uso de $\operatorname{lir}^{e} \operatorname{son}^{e} k e m$ ("de modo que sean aceptadas en favor de ustedes") dondequiera que es ordenado en Levítico; y segundo, por ser ejecutado solo después de los demás sacrificios (Lv 9:22). ${ }^{30}$ En otras palabras, un sacrificio de paz aceptado por Dios llegaba a ser un beneficio para el adorador siempre y cuando éste lo ofreciera siguiendo las ordenanzas rituales estatuidas en la ley levítica, lo cual implicaba sacrificar el animal, compartirlo y consumirlo dentro del tiempo adecuado. Además, debido a que comúnmente se realizaban primero el resto de sacrificios cruentos, la repetición del procedimiento sacrificial tenía un propósito nítidamente conmemorativo del precio de su perdón: la sangre derramada. ${ }^{31}$

De esta manera, el sacrificio de paz era realizado una vez que el

${ }^{29}$ Elementos importantes semejantes a los demás sacrificios eran: la imposición de manos sobre el animal, y el derramamiento de sangre alrededor del altar. Dado que los sacrificios de paz no son descritos en ninguna parte como cumpliendo una función expiatoria, ciertamente tenían el propósito de hacer recordar al creyente lo que costó su estado de paz actual: el derramamiento de sangre. Incluso se ha sugerido que su orden postrero al momento de ejecutar los sacrificios tiene cierta relación con Efesios 2:14, donde se afirma que Cristo "es nuestra paz". Ver G. Lloyd Carr, "שָׁׁ", en TWOT, 932.

${ }^{30}$ Averbeck, 715 .

${ }^{31} \mathrm{El}$ motivo de la sangre derramada en el Pentateuco permite percibir la significancia de este evento. El asesinato tiene implicaciones extensas, que van desde la culpabilidad misma del asesino, hasta la contaminación espacial del territorio donde este mora. Ver Karl G. Boskamp, "El motivo de la sangre derramada en el Pentateuco", DavarLogos 13/1 (2014): 21-35. Trasladando este concepto al ámbito del santuario, puede deducirse que el sacrificio de paz instilaba estas nociones en la mente del oferente, razón por la cual éste se encontraría plenamente agradecido pues su culpa ya había sido quitada gracias al sacrificio vicario figurativo del animal. 
adorador tuviera conciencia de que su relación con Dios había sido restaurada por la expiación realizada en su favor en el santuario. Además, al seguir un procedimiento similar al de los demás sacrificios, tal persona rememoraba lo que logró su estado actual de paz: un derramamiento de sangre (Heb 9:22). Considerando que a esto se sumaban las razones que motivaban el sacrificio de paz y la participación de Jehová, del sacerdote y de los amigos del oferente, queda definido lo singular y gozoso de la ocasión. Dicho de otro modo, un sacrificio de paz era ofrecido en un estado de comunión y paz con Dios y los semejantes en la vida del adorador, siendo una expresión completamente voluntaria, producida por lo que Dios ya había hecho por la persona.

\section{Conclusión}

Han pasado más de $3460^{32}$ años desde que se establecieron estos principios en la iglesia del pasado. No obstante, su divino Autor los dio para que puedan extraerse lecciones valiosas para la fidelidad de la iglesia moderna, tales como:

1. Si bien se puede expresar gratitud, confesión, alabanza, compromiso y buena voluntad para con Dios mediante palabras y emociones, se requiere un "sacrificio de paz", una ofrenda externa que brote del corazón.

2. La práctica cristiana más vulnerable a impersonalizarse es la de dar ofrendas. Con los años, llega a ser una práctica mecánica, desprovista de toda noción de relación con Dios y reverencia a Él. Las ofrendas son dirigidas a un Dios de amor y, a

${ }^{32}$ Hoy es posible calcular esta cifra merced al desarrollo de la cronología bíblica. De acuerdo a la afirmación de 1 de Reyes 6:1, se determina que el Éxodo debe haber tenido lugar en 1446 a.C. Debido a que las instrucciones consignadas en el libro de Levítico fueron dadas luego del "día primero del primer mes, en el segundo año" (Éx 40:17), indubitablemente fueron dadas a Moisés en el año 1445 a.C. Ver Finegan, 201. 
la vez, a un Dios santo quien es dueño aún de la vida humana. Por eso, él requiere todo el corazón (Dt 6:5).

3. Una ofrenda aceptable a Dios es la que se realiza de acuerdo con lo prescrito por Él. Cuando el creyente sigue por fe las ordenanzas divinas experimenta el poder vivificador que emana de Dios (Lv 18:5). La norma que se ha de seguir en estos días es la misma que la que siguieron los apóstoles (1 Cor 16:1).

4. Finalmente, una ofrenda aceptable es la que es ofrecida recordando lo que Dios ha hecho, hace y hará en la vida del creyente. No se trata de una contribución caritativa o una limosna desprovista de toda devoción. Por otro lado, al momento de ofrendar "la sinceridad del propósito y la bondad genuina del corazón son los motivos apreciados por el cielo". ${ }^{33}$

Sin duda, el mayor motivo de gratitud y gozo para el creyente es la redención lograda en favor de la humanidad. Así como al ofrecer un sacrificio de paz, dar ofrendas en estos días se realiza por lo que Dios ya hizo por los fieles llegando a ser una bendición para los mismos fieles ( $l i$ r'șononkem), pues dada está la promesa: "La paz os dejo, mi paz os doy; yo no os la doy como el mundo la da" (Jn 14:27).

Recibido: $27 / 12 / 15$

Aceptado: 03/02/16

${ }^{33}$ Elena G. de White, Consejos sobre mayordomía cristiana (Buenos Aires: Asociación Casa Editora Sudamericana, 2007), 125. 